\title{
Quantum field theory of van der Waals friction
}

\author{
A. I. Volokitin ${ }^{1,2, *}$ and B. N. J. Persson ${ }^{1}$ \\ ${ }^{1}$ Institut für Festkörperforschung, Forschungszentrum Jülich, D-52425, Germany \\ ${ }^{2}$ Samara State Technical University, 443100 Samara, Russia \\ (Received 17 May 2006; revised manuscript received 15 September 2006; published 13 November 2006)

\begin{abstract}
van der Waals friction between two semi-infinite solids, and between a small neutral particle and semiinfinite solid is studied using thermal quantum field theory in the Matsubara formulation. We show that the friction to linear order in the sliding velocity can be obtained from the equilibrium Green functions and that our treatment can be extended for bodies with complex geometry. The calculated friction agrees with the friction obtained using a dynamical modification of the Lifshitz theory, which is based on the fluctuation-dissipation theorem. We show that it should be possible to measure the van der Waals friction in noncontact friction experiment using state-of-the-art equipment.
\end{abstract}

DOI: 10.1103/PhysRevB.74.205413

PACS number(s): 11.10.Wx, 12.20.-m, 42.50.Lc

\section{INTRODUCTION}

A great deal of attention has been devoted to the problem of noncontact friction between nanostructures, including, for example, the frictional drag force between electrons in twodimensional quantum wells ${ }^{1-3}$ and the friction force between an atomic force microscope tip and a substrate. ${ }^{4-13}$ The interest in noncontact friction is, at least in part, because of the importance of noncontact friction for ultrasensitive force detection experiments. The ability to detect small forces is inextricably linked to friction via the fluctuation-dissipation theorem. According to this theorem, the random force that makes a small particle jitter also cause friction if the particle is dragged through the medium. The detection of single spins by magnetic resonance force microscopy ${ }_{14}{ }^{14}$ which has been proposed for three-dimensional atomic imaging ${ }^{15}$ and quantum computation, ${ }^{16}$ will require force fluctuations (and consequently the friction) to be reduced to unprecedented levels. In addition, the search for quantum gravitation effects at short length scale, ${ }^{17}$ and future measurements of the Casimir and van der Waals forces, ${ }^{18}$ may eventually be limited by noncontact friction effects.

In noncontact friction the bodies are separated by a potential barrier thick enough to prevent electrons or other particles with a finite rest mass from tunneling across it, but allowing interaction via the long-range electromagnetic field, which is always present in the gap between bodies. The presence of an inhomogeneous tip-sample electric field is difficult to avoid, even under the best experimental conditions. ${ }^{6}$ For example, even if both the tip and the sample were metallic single crystals, the tip would still have corners and more than one crystallographic plane exposed. The presence of atomic steps, adsorbates, and other defects will also contribute to the spatial variation of the surface potential. This is referred to as the "patch effect." The surface potential can also be easily changed by applying a voltage between the tip and the sample. An inhomogeneous electric field can also be created by charged defects embedded in a dielectric sample. The relative motion of the charged bodies will produce friction which is denoted as "electrostatic friction."

The electromagnetic field can also be created by the fluctuating current density due to thermal and quantum fluctua- tions inside the bodies. This fluctuating electromagnetic field gives rise to the well-known long-range attractive van der Waals interaction between two bodies ${ }^{19,20}$ and is responsible for radiative heat transfer. If the bodies are in relative motion, the same fluctuating electromagnetic field will give rise to a friction which is denoted as "Van der Waals friction."

The origin of van der Waals friction is closely connected with the van der Waals interaction. The van der Waals interaction arises when an atom or molecule spontaneously develops an electric dipole moment due to quantum fluctuations. The short-lived atomic polarity can induce a dipole moment in a neighboring atom or molecule some distance away. The same is true for extended media, where thermal and quantum fluctuation of the current density in one body induces a current density in the other body; the interaction between these current densities is the origin of the van der Waals interaction. When two bodies are in relative motion, the induced current will lag slightly behind the fluctuating current inducing it, and this is the origin of van der Waals friction.

The van der Waals interaction is mainly determined by exchange of virtual photons between the bodies (connected with quantum fluctuations), and does not vanish even at zero temperature. On the other hand, van der Waals friction, at least to lowest order of perturbation theory, and to linear order in the sliding velocity, is determined by exchange of real photons, and vanishes at zero temperature.

To clarify the origin of van der Waals friction let us consider two flat parallel surfaces, separated by a sufficiently wide vacuum gap, which prevents electrons from tunneling across it. If the surfaces are in relative motion (velocity $v$ ) a frictional stress will act between them. This frictional stress is related with an asymmetry of the reflection coefficient along the direction of motion; see Fig. 1. If one body emits radiation, then in the rest reference frame of the second body these waves are Doppler shifted which will result in different reflection coefficients. The same is true for radiation emitted by the second body. The exchange of "Doppler shifted photons" is the origin of van der Waals friction.

The van der Waals friction originates from two types of processes. (a) Photons are created in each body with opposite momentum and the frequencies of these photons are connected by $v q_{x}=\omega_{1}+\omega_{2}$, where $q_{x}$ is the momentum transfer. 


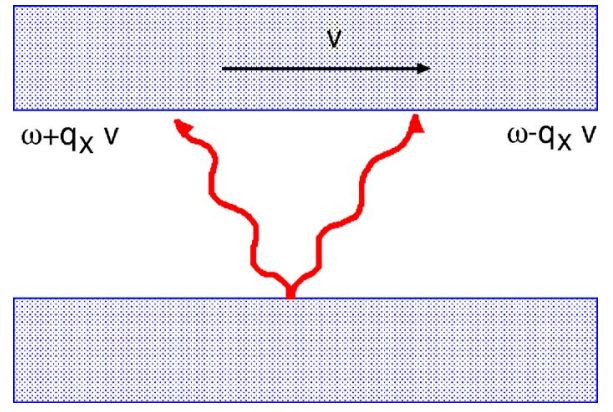

FIG. 1. (Color online) The electromagnetic waves emitted in the opposite direction by the body at the bottom will experience opposite Doppler shift in the reference frame in which the body at the top is at rest. Due to the frequency dispersion of the reflection coefficient these electromagnetic waves will reflect differently from the surface of the body at the top, which give rises to momentum transfer between the bodies. This momentum transfer is the origin of van der Waals friction.

(b) A photon is annihilated in one body and created in another. The first process (a) is possible even at zero temperature, and gives rise to a friction force which depends cubically on sliding velocity. ${ }^{21,22}$ The second process (b) is possible only at finite temperatures, and gives rise to a friction which depends linearly on the sliding velocity. Thus, process (b) will give the main contribution to the friction at sufficiently high temperatures, and at not too large velocities.

In contrast to the van der Waals interaction, for which theory is well established, the van der Waals friction is still controversial topic. As an example, different authors have studied the van der Waals friction between two flat surfaces in parallel relative motion using different methods, and obtained results which are in sharp contradiction to each other. The first calculation of the van der Waals friction was done by Teodorovich. ${ }^{23}$ Teodorovich assumed that the force of friction can be calculated as the ordinary van der Waals force between bodies at rest, whose dielectric function depend on the velocity due to the Doppler shift. However, from the dynamical modification of the Lifshitz's theory it follows ${ }^{22}$ that it is not true. Later the same approach was used by Mahanty ${ }^{24}$ to calculate the friction between molecules. Both theories predict wrong nonzero friction at zero temperature and to linear order in the sliding velocity. The same nonzero linear friction at zero temperature was predicted in Refs. 25 and 26. In Ref. 27 it was shown that the basic equation in Refs. 25 and 26 is incorrect, and a correct treatment gives a friction which, to linear order in the sliding velocity, vanishes at $T=0 \mathrm{~K}$. Schaich and Harris developed a theory ${ }^{28}$ which describes the dependence of friction on the temperature $T$ and on the separation $d$. However in their calculations they made some unphysical approximations, and as a result they did not get the correct final formula for the friction for parallel relative motion. For normal relative motion they considered only the case of zero temperature where, to linear order in the sliding velocity, the friction is determined by higher order processes related with the renormalization of the electron self-energy. The friction obtained in Refs. 29-31 vanishes in the limit of infinite light velocity $c \rightarrow \infty$. However, at least for short distances, one can neglect retardation effects when calculating the van der Waals friction (as for the van der Waals interaction). Pendry ${ }^{21}$ assumed zero temperature and neglected retardation effects, in which case the friction depends cubically on the velocity. Persson and Zhang ${ }^{32}$ obtained the formula for friction in the limit of small velocities and finite temperature using a simple quantum mechanical approach, again neglecting retardation effects. In Ref. 27 Volokitin and Persson developed a theory of the van der Waals friction based on the dynamical modification of the well known Lifshitz theory ${ }^{19}$ of the van der Waals interaction. In the nonretarded limit and for zero temperature this theory agrees with the results of Pendry. ${ }^{21}$ Similarly, in the nonretarded limit and for small sliding velocity this theory agrees with the study of Persson and Zhang. ${ }^{32}$

In Refs. 9 and 10 the theory was extended to two flat surfaces in normal relative motion. For resonant photon tunneling between surface localized states, normal relative motion results in a different result as for parallel relative motion. It was shown that the friction may increase by many orders of magnitude when the surfaces are covered by adsorbates, or can support low-frequency surface plasmons Refs. 9 and 10. In this case the friction is determined by resonant photon tunneling between adsorbate vibrational modes, or surface plasmon modes. When one of the bodies is sufficiently rarefied, this theory Refs. 9 and 10 gives the friction between a flat surface and a small particle, which in the nonretarded limit agrees with the results of Tomassone and Widom. ${ }^{33} \mathrm{~A}$ theory of the van der Waals friction between a small particle and flat surface, which takes into account screening, nonlocal optic effects, and retardation effects, was developed in Ref. 27.

At present there are many theories of the van der Waals friction, which frequently contradict each other, but a rigorous theory based on quantum field theory has not been presented. A quantum field theory for the van der Waals interaction was developed in Ref. 20, where the van der Waals stress tensor was expressed in terms of finite-temperature Green's functions. This theory can be applied to some problem which cannot be solved using Lifshitz theory of the van der Waals interaction..$^{20}$ In particular, this approach was used in Ref. 34 to obtain the van der Waals force between a sphere and a semi-infinite slab. Due to the great importance of the van der Waals friction for understanding the origin of noncontact friction, in this article we develop a rigorous theory of the van der Waals friction based on quantum field theory.

This article is organized as follows. In Sec. II we present a short overview of the basic idea of the quantum field theory of the van der Waals friction. We show how to obtain the van der Waals friction in terms of the finite-temperature Green's functions of the electromagnetic field. As applications we derived the van der Waals friction between two semi-infinite solids (Sec. III), and between a small particle and a semiinfinite solid (Sec. IV), for both parallel and normal relative motion. These calculations confirm early results obtained using he dynamical modification of the Lifshitz theory and the fluctuation-dissipation theorem, and shows that the Lifshitz theory can be applied to bodies in relative motion. However, the quantum field theory is more general and can be applied for bodies with complex geometry. In Sec. V we show that the van der Waals friction can be greatly enhanced for high- 
resistivity metals, for dielectrics with strong absorption at low frequencies, and for two-dimensional (2D) systems, e.g., $2 \mathrm{D}$-electron systems on dielectric substrates, or incommensurate layers of adsorbed ions exhibiting acoustic vibrations. Section VI presents the conclusions and the outlook. Appendixes $\mathrm{A}$ and $\mathrm{B}$ contain some details of the derivations of the Green functions of the electromagnetic field used in Secs. III and IV.

\section{GENERAL FORMALISM}

There are two approaches to the theories of the van der Waals interaction and the van der Waals friction. In the first approach the fluctuating electromagnetic field is considered as a classical field which can be calculated from Maxwell's equations with the fluctuating current density as the source of the field, and with appropriate boundary conditions. This approach was used by Lifshitz in the theory of the van der Waals interaction ${ }^{19}$ and by Volokitin and Persson for the van der Waals friction. ${ }^{10,22}$ The calculation of the van der Waals friction is more complicated than of the van der Waals force because it requires the determination of the electromagnetic field between moving boundaries. The solution can be found by writing the boundary conditions on the surface of each body in the rest reference frame of this body. The relation between the electromagnetic fields in the different reference frames is determined by the Lorenz transformation. The advantage of this approach is in that, in principle, it can be used for the calculation of friction for arbitrary relative velocities. However, the calculations become very complicated for bodies with complex geometry, and the solutions are only known for two parallel plane surfaces, ${ }^{10,22}$ and between a small particle and plane surface. ${ }^{27}$

In the second approach the electromagnetic field is treated in the frame of the quantum field theory ${ }^{35}$ This approach was used in Ref. 20 to obtain the van der Waals interaction for an arbitrary inhomogeneous medium all parts of which are at rest. Here we will use a similar approach to calculate the van der Waals friction.

For two bodies in slow uniform relative motion (velocity $\mathbf{v}$ ) the force acting on either body may be written as $\mathbf{F}=\mathbf{F}_{0}$ $-\stackrel{\leftrightarrow}{\Gamma} \cdot \mathbf{v}$, where the adiabatic force $\mathbf{F}_{0}$ is independent of $\mathbf{v}$, and $\leftrightarrow$

$\boldsymbol{\Gamma}$, the so-called friction tensor, is defined by

$$
\stackrel{\leftrightarrow}{\boldsymbol{\Gamma}}=\left(k_{B} T\right)^{-1} \operatorname{Re} \int_{0}^{\infty} d t\langle\hat{\mathbf{F}}(t) \hat{\mathbf{F}}(0)\rangle .
$$

Here $\langle\cdots\rangle$ represents thermal average of the fluctuating force in the equilibrium state at fixed separation $d$ between the bodies, and $\hat{\mathbf{F}}(t)$ is the force operator in the Heisenberg representation. Equation (1) is a consequence of the fluctuationdissipation theorem. ${ }^{36}$ For the interaction between a localized and an extended system, Eq. (1) has been derived by several authors (Schaich, ${ }^{37}$ d'Agliano et al. ${ }^{38}$ Nourtier $^{39}$ ) and is also valid for two extended systems. In the context of the van der Waals friction Eq. (1) was used by Schaich and Harris, ${ }^{28}$ but their treatment is incomplete.
In the case of extended systems the fluctuating force operator can be expressed through the operator of the stress tensor $\hat{\sigma}_{i k}$

$$
\hat{F}_{i}=\int d S_{k} \hat{\sigma}_{i k}
$$

where the integration is over the surface of one of the bodies and

$$
\hat{\sigma}_{i k}=\frac{1}{8 \pi}\left[E_{i} E_{k}+E_{k} E_{i}+B_{i} B_{k}+B_{k} B_{i}-\delta_{i k}\left(E^{2}+B^{2}\right)\right],
$$

where $E_{i}$ and $B_{i}$ are the electric and magnetic induction field operator, respectively. The calculation of the force-force correlation function can be done using the methods of the quantum field theory. ${ }^{35,40}$ Such calculations are described in Sec. III for two plane parallel surfaces, and in Sec. IV for a small particle and plane surface, for both parallel and normal relative motion. The advantage of this approach is that it only involves finding of the Green's functions of the electromagnetic field for the equilibrium system with fixed boundaries. Thus, this approach can be easily extended to bodies with complex geometry. However, it is restricted to small relative velocities.

\section{VAN DER WAALS FRICTION BETWEEN TWO PLANE SURFACE}

\section{A. Parallel relative motion}

Assume that the $x y$ plane coincides with one of the surfaces. For parallel relative motion the friction coefficient $\Gamma_{\|}$ $=\Gamma_{x x}=\Gamma_{y y}$. Using the methods of quantum field theory ${ }^{35}$ the expression for the friction coefficient (1) for parallel relative motion can be written in the form

$$
\Gamma_{\|}=\lim _{\omega_{0} \rightarrow 0} \operatorname{Im} \frac{G_{x x}^{R}\left(\omega_{0}+i \delta\right)}{\omega_{0}},
$$

where $G_{x x}^{R}$ is the retarded Green's function determined by

$$
G_{x x}^{R}(\omega)=\frac{i}{\hbar} \int_{0}^{\infty} d t e^{i \omega t}\left\langle\hat{F}_{x}(t) \hat{F}_{x}(0)-\hat{F}_{x}(0) \hat{F}_{x}(t)\right\rangle,
$$

where

$$
\hat{F}_{x}=\int d S_{z} \hat{\sigma}_{x z}
$$

where the surface integral is taken over the surface of the body at $z=0$,

$$
\hat{\sigma}_{x z}=\left(E_{x} E_{z}+E_{z} E_{x}+B_{x} B_{z}+B_{z} B_{x}\right) / 8 \pi .
$$

The function $G_{x x}^{R}$ can be obtained by analytic continuation in the upper half of $\omega$ plane of the temperature Green's function $G_{x x}(\omega)$, determined on the discrete set of point $i \omega_{n}$ $=i 2 \pi n / \beta$ by the formula

$$
G_{x x}\left(i \omega_{n}\right)=-\frac{1}{\hbar} \int_{0}^{\beta} d \tau e^{i \omega_{n} \tau}\left\langle T_{\tau} \hat{F}_{x}(\tau) \hat{F}_{x}(0)\right\rangle,
$$

where $n$ is an integer and $\beta=\hbar / k_{B} T . T_{\tau}$ is the time-ordering operator. The function $G_{x x}\left(i \omega_{n}\right)$ can be calculated using stan- 
dard techniques of quantum field theory ${ }^{35,40}$ and can be represented through the Green's functions of the electromagnetic field

$$
\begin{aligned}
D_{i j}^{E E}\left(\mathbf{r}, \mathbf{r}^{\prime}, i \omega_{n}\right) & =D_{i j}\left(\mathbf{r}, \mathbf{r}^{\prime}, i \omega_{n}\right) \\
& =-\frac{1}{\hbar} \int_{0}^{\beta} d \tau e^{i \omega_{n} \tau}\left\langle T_{\tau} \hat{E}_{i}(\tau) \hat{E}_{j}(0)\right\rangle,
\end{aligned}
$$

where the retarded Green functions $D_{i j}\left(\mathbf{r}, \mathbf{r}^{\prime}, \omega\right)$ obey the equations $^{35}$

$$
\begin{aligned}
&\left(\boldsymbol{\nabla}_{i} \boldsymbol{\nabla}_{k}-\delta_{i k} \nabla^{2}\right) D_{k j}\left(\mathbf{r}, \mathbf{r}^{\prime}, \omega\right) \\
&-(\omega / c)^{2} \int d^{3} x^{\prime \prime} \epsilon_{i k}\left(\mathbf{r}, \mathbf{r}^{\prime \prime}, \omega\right) D_{k j}\left(\mathbf{r}^{\prime \prime}, \mathbf{r}^{\prime}, \omega\right) \\
&=\left(4 \pi \omega^{2} / c^{2}\right) \delta_{i j} \delta\left(\mathbf{r}-\mathbf{r}^{\prime}\right), \\
&\left(\boldsymbol{\nabla}_{j}^{\prime} \nabla_{k}^{\prime}-\delta_{j k} \nabla^{\prime 2}\right) D_{i k}\left(\mathbf{r}, \mathbf{r}^{\prime}, \omega\right) \\
& \quad-(\omega / c)^{2} \int d^{3} x^{\prime \prime} \epsilon_{k j}\left(\mathbf{r}^{\prime \prime}, \mathbf{r}^{\prime}, \omega\right) D_{i k}\left(\mathbf{r}, \mathbf{r}^{\prime \prime}, \omega\right) \\
&=\left(4 \pi \omega^{2} / c^{2}\right) \delta_{i j} \delta\left(\mathbf{r}-\mathbf{r}^{\prime}\right) .
\end{aligned}
$$

For the plane surface it is convenient to decompose the electromagnetic field into $s$ - and $p$-polarized plane waves. Introducing $\hat{q}=\mathbf{q} / q$ and $\hat{n}=[\hat{z} \times \hat{q}]$, where $\mathbf{q}$ is the surface component of the wave vector, the Green's tensor is given by

$$
\begin{aligned}
\stackrel{\leftrightarrow}{\mathbf{D}^{E E}\left(\mathbf{r}, \mathbf{r}^{\prime}\right)=} & \int \frac{d^{2} q}{(2 \pi)^{2}}\left[\hat{n} D_{n n}^{E E}\left(z, z^{\prime}, \mathbf{q}\right) \hat{n}+\hat{q} D_{q q}^{E E}\left(z, z^{\prime}, \mathbf{q}\right) \hat{q}\right. \\
& +\hat{z} D_{z z}^{E E}\left(z, z^{\prime}, \mathbf{q}\right) \hat{z}+\hat{z} D_{z q}^{E E}\left(z, z^{\prime}, \mathbf{q}\right) \hat{q} \\
& \left.+\hat{q} D_{q z}^{E E}\left(z, z^{\prime}, \mathbf{q}\right) \hat{z}\right] \mathbf{e}^{i \mathbf{q} \cdot\left(\mathbf{x}-\mathbf{x}^{\prime}\right)},
\end{aligned}
$$

where we have taken into account that $D_{n z}^{E E}=D_{n q}^{E E}=0$ (see Appendix A). For two plane parallel surfaces the solution of Eqs. (10), (11) is derived in Appendix A. Using the methods of the quantum field theory ${ }^{35,40}$ for the Green function $G_{x x}$ we get

$$
\begin{aligned}
G_{x x}\left(i \omega_{n}\right)= & \frac{\hbar A}{16 \pi^{2} \beta} \int \frac{d^{2} \mathbf{q}}{(2 \pi)^{2}} \sum_{\omega_{m}} \frac{q_{x}^{2}}{q^{2}}\left[D_{q q}^{E E}\left(\mathbf{q}, i \omega_{m}, z, z^{\prime}\right)\right. \\
& \times D_{z z}^{E E}\left(-\mathbf{q}, i \omega_{n}-i \omega_{m}, z, z^{\prime}\right)+D_{q z}^{E E}\left(\mathbf{q}, i \omega_{m}, z, z^{\prime}\right) \\
& \times D_{z q}^{E E}\left(-\mathbf{q}, i \omega_{n}-i \omega_{m}, z, z^{\prime}\right)+D_{q q}^{B B}\left(\mathbf{q}, i \omega_{m}, z, z^{\prime}\right) \\
& \times D_{z z}^{B B}\left(-\mathbf{q}, i \omega_{n}-i \omega_{m}, z, z^{\prime}\right)+D_{q z}^{B B}\left(\mathbf{q}, i \omega_{m}, z, z^{\prime}\right) \\
& \left.\times D_{z q}^{B B}\left(-\mathbf{q}, i \omega_{n}-i \omega_{m}, z, z^{\prime}\right)\right]_{z=z^{\prime}=0},
\end{aligned}
$$

where $A$ is the surface area, and $D_{i j}^{B B}\left(\mathbf{q}, i \omega_{m}, z, z^{\prime}\right)$ is given by ${ }^{35}$

$$
D_{i j}^{B B}\left(\mathbf{r}, \mathbf{r}^{\prime}, i \omega_{n}\right)=-\left(\frac{c}{\omega_{n}}\right)^{2} e_{i l k} e_{j s t} \nabla_{l} \nabla_{s}^{\prime} D_{k t}^{E E}\left(\mathbf{r}, \mathbf{r}^{\prime}, i \omega_{n}\right),
$$

where $e_{i j l}$ is the completely asymmetric unit tensor. In Eq. (13) we omitted terms involving product of Green's functions associated with the $p$ - and $s$-polarized electromagnetic field because, after the frequency summations, they cancel each other. Even without any detailed calculations it is clear that such terms must give zero contribution to the friction because the $p$ - and $s$-polarized waves must give independent contributions to the friction.

Performing the frequency summations in Eq. (13) (see Appendix B) gives

$$
\begin{aligned}
\gamma_{x x}= & \frac{\hbar}{8 \pi^{3}} \int_{0}^{\infty} d \omega\left(-\frac{\partial n}{\partial \omega}\right) \int \frac{d^{2} \mathbf{q}}{(2 \pi)^{2}} \frac{q_{x}^{2}}{q^{2}}\left\{\left[\operatorname{Im} D_{q q} \operatorname{Im} D_{z z}\right.\right. \\
& \left.-\frac{q^{2}}{\gamma^{4}}\left(\frac{\partial}{\partial z} \operatorname{Im} D_{q q}\right)\left(\frac{\partial}{\partial z^{\prime}} \operatorname{Im} D_{q q}\right)\right] \\
& +\left(\frac{c}{\omega}\right)^{4} q^{2}\left[\operatorname{Im} D_{n n} \frac{\partial^{2}}{\partial z \partial z^{\prime}} \operatorname{Im} D_{n n}\right. \\
& \left.\left.-\left(\frac{\partial}{\partial z} \operatorname{Im} D_{n n}\right)\left(\frac{\partial}{\partial z^{\prime}} \operatorname{Im} D_{n n}\right)\right]\right\}_{z=z^{\prime}=0},
\end{aligned}
$$

where $n(\omega)=\left[\exp \left(\hbar \omega / k_{B} T\right)-1\right]^{-1}$. Using Eqs. (A9), (A13), (A15) for the Green's functions in Eq. (15), the contribution to the friction from the propagating $(q<\omega / c)$ waves becomes

$$
\begin{aligned}
\gamma_{\|}^{\mathrm{rad}}= & \frac{\hbar}{8 \pi^{3}} \int_{0}^{\infty} d \omega\left(-\frac{\partial n}{\partial \omega}\right) \int_{q \omega / c} d^{2} \mathbf{q} q_{x}^{2} \times\left[\operatorname{Re}\left(\frac{1+R_{1 p} R_{2 p} e^{2 i \gamma d}-R_{1 p}-R_{2 p} e^{2 i \gamma d}}{1-e^{2 i \gamma d} R_{1 p} R_{2 p}}\right) \operatorname{Re}\left(\frac{1+R_{1 p} R_{2 p} e^{2 i \gamma d}+R_{1 p}+R_{2 p} e^{2 i \gamma d}}{1-e^{2 i \gamma d} R_{1 p} R_{2 p}}\right)\right. \\
& \left.-\left(\operatorname{Im} \frac{R_{1 p}-R_{2 p} e^{2 i \gamma d}}{1-e^{2 i \gamma d} R_{1 p} R_{2 p}}\right)^{2}+[p \rightarrow s]\right]=\frac{\hbar}{8 \pi^{2}} \int_{0}^{\infty} d \omega\left(-\frac{\partial n}{\partial \omega}\right) \int_{0}^{\omega / c} d q q^{3} \frac{\left(1-\left|R_{1 p}\right|^{2}\right)\left(1-\left|R_{2 p}\right|^{2}\right)}{\left|1-e^{2 i \gamma d} R_{1 p} R_{2 p}\right|^{2}}+[p \rightarrow s] .
\end{aligned}
$$

where the symbols $[p \rightarrow s]$ denotes the term which is obtained from the first one by replacement of the reflection amplitude $R_{p}$ for $p$-polarized waves by the reflection amplitude $R_{s}$ for $s$ - polarized waves. Similarly, the contribution to the friction from the evanescent electromagnetic waves $(q>\omega / c)$ : 


$$
\begin{aligned}
\gamma_{\|}^{\mathrm{evan}}= & \frac{\hbar}{8 \pi^{3}} \int_{0}^{\infty} d \omega\left(-\frac{\partial n}{\partial \omega}\right) \int_{q<\omega / c} d^{2} \mathbf{q} q_{x}^{2} \times\left[-\operatorname{Im}\left(\frac{2 R_{1 p} R_{2 p} e^{-2|\gamma| d}-R_{1 p}-R_{2 p} e^{-2|\gamma| d}}{1-e^{-2|\gamma| d} R_{1 p} R_{2 p}}\right) \times \operatorname{Im}\left(\frac{2 R_{1 p} R_{2 p} e^{-2|\gamma| d}+R_{1 p}+R_{2 p} e^{-2|\gamma| d}}{1-e^{-2|\gamma| d} R_{1 p} R_{2 p}}\right)\right. \\
& \left.-\left(\operatorname{Im} \frac{R_{1 p}-R_{2 p} e^{-2|\gamma| d}}{1-e^{-2|\gamma| d} R_{1 p} R_{2 p}}\right)^{2}+[p \rightarrow s]\right]=\frac{\hbar}{2 \pi^{2}} \int_{0}^{\infty} d \omega\left(-\frac{\partial n}{\partial \omega}\right) \int_{\omega / c}^{\infty} d q q^{3} e^{-2|\gamma| d} \frac{\operatorname{Im} R_{1 p} \operatorname{Im} R_{2 p}}{\left|1-e^{-2|\gamma| d} R_{1 p} R_{2 p}\right|^{2}}+[p \rightarrow s] .
\end{aligned}
$$

Equations (16) and (17) were first derived in Ref. 22 using the dynamical modification of the Lifshitz theory.

\section{B. Normal relative motion}

For two plane surfaces in normal relative motion the force operator is given by

$$
\hat{F}_{z}=\int d S_{z} \hat{\sigma}_{z z}
$$

where

$$
\hat{\sigma}_{z z}=\left(E_{z} E_{z}-E_{x} E_{x}-E_{y} E_{y}+B_{z} B_{z}-B_{x} B_{x}-B_{y} B_{y}\right) / 8 \pi .
$$

The friction coefficient for normal relative motion can be obtained from the analytical continuation in the upper part of $\omega$ plane of the Green function $G_{z z}\left(i \omega_{n}\right)$ which is determined by

$$
\begin{aligned}
G_{z z}\left(i \omega_{n}\right)= & \frac{\hbar A}{32 \pi^{2} \beta} \int \frac{d^{2} \mathbf{q}}{(2 \pi)^{2}} \sum_{\omega_{m}} \frac{q_{x}^{2}}{q^{2}}\left[D_{z z}^{E E} D_{z z}^{E E}+D_{q q}^{E E} D_{q q}^{E E}\right. \\
& +D_{n n}^{E E} D_{n n}^{E E}-D_{z q}^{E E} D_{z q}^{E E}-D_{q z}^{E E} D_{q z}^{E E}-D_{z n}^{E B} D_{z n}^{E B} \\
& \left.-D_{n z}^{E B} D_{n z}^{E B}+D_{q n}^{E B} D_{q n}^{E B}+D_{n q}^{E B} D_{n q}^{E B}+[E \leftrightarrow B]\right],
\end{aligned}
$$

where the arguments of the Green functions in Eq. (20) are the same as in Eq. (13), $[E \leftrightarrow B]$ denotes the terms which can be obtained from the first terms by permutation of the upper case indexes $E$ and $B$ and

$$
\begin{gathered}
D_{i j}^{E B}\left(\mathbf{r}, \mathbf{r}^{\prime}, \omega_{n}\right)=\frac{c}{\omega_{n}} e_{j k l} \nabla_{k}^{\prime} D_{i l}^{E E}\left(\mathbf{r}, \mathbf{r}^{\prime}, \omega_{n}\right), \\
D_{i j}^{B E}\left(\mathbf{r}, \mathbf{r}^{\prime}, \omega_{n}\right)=-\frac{c}{\omega_{n}} e_{i k l} \nabla_{k} D_{l j}^{E E}\left(\mathbf{r}, \mathbf{r}^{\prime}, \omega_{n}\right) .
\end{gathered}
$$

Performing similar calculations as for the parallel relative motion we get

$$
\begin{aligned}
\gamma_{\perp}= & \frac{\hbar}{16 \pi^{3}} \int_{0}^{\infty} d \omega\left(-\frac{\partial n}{\partial \omega}\right) \int \frac{d^{2} \mathbf{q}}{(2 \pi)^{2}} \times\left\{\left[\left(\operatorname{Im} D_{q q}\right)^{2}+\frac{\gamma^{4}}{q^{4}}\left(\operatorname{Im} D_{z z}\right)^{2}+\frac{2}{\gamma^{2}}\left(\frac{\partial}{\partial z} \operatorname{Im} D_{z z}\right)^{2}\right]+\left(\frac{c}{\omega}\right)^{4}\left[\gamma^{4}\left(\operatorname{Im} D_{n n}\right)^{2}\right.\right. \\
& \left.\left.+\left(\frac{\partial^{2}}{\partial z \partial z^{\prime}} \operatorname{Im} D_{n n}\right)^{2}+2 \gamma^{2}\left(\frac{\partial}{\partial z^{\prime}} \operatorname{Im} D_{n n}\left(z, z^{\prime}\right)\right)^{2}\right]\right\}_{z=z^{\prime}=0} \cdot
\end{aligned}
$$

Substitution the expressions for the Green's functions from Eqs. (A9), (A13), (A15) in Eq. (23) gives the contribution to the friction from the propagating waves

$$
\begin{aligned}
\gamma_{\perp}^{a d}= & \frac{\hbar}{16 \pi^{3}} \int_{0}^{\infty} d \omega\left(-\frac{\partial n}{\partial \omega}\right) \int_{q<\omega / c} d^{2} \mathbf{q} \gamma^{2} \times\left[\left(\operatorname{Re} \frac{1+R_{1 p} R_{2 p} e^{2 i \gamma d}-R_{1 p}-R_{2 p} e^{2 i \gamma d}}{1-e^{2 i \gamma d} R_{1 p} R_{2 p}}\right)^{2}+\left(\operatorname{Re} \frac{1+R_{1 p} R_{2 p} e^{2 i \gamma d}+R_{1 p}+R_{2 p} e^{2 i \gamma d}}{1-e^{2 i \gamma d} R_{1 p} R_{2 p}}\right)^{2}\right. \\
& \left.+2\left(\operatorname{Im} \frac{R_{1 p}-R_{2 p} e^{2 i \gamma d}}{1-e^{2 i \gamma d} R_{1 p} R_{2 p}}\right)^{2}+[p \rightarrow s]\right]=\frac{\hbar}{4 \pi^{2}} \int_{0}^{\infty} d \omega\left(-\frac{\partial n}{\partial \omega}\right) \int_{0}^{\omega / c} d q q \gamma^{2} \\
& \times \frac{\left(1-\left|R_{1 p}\right|^{2}\left|R_{2 p}\right|^{2}\right)^{2}+\left|\left(1-\left|R_{1 p}\right|^{2}\right) R_{2 p} e^{i \gamma d}+\left(1-\left|R_{2 p}\right|^{2}\right) R_{1 p}^{*} e^{-i \gamma d}\right|^{2}}{\left|1-e^{2 i \gamma d} R_{1 p} R_{2 p}\right|^{4}}+[p \rightarrow s]
\end{aligned}
$$

In a similar way one can obtain the contribution to the friction from the evanescent electromagnetic waves 


$$
\begin{aligned}
\gamma_{\perp}^{\text {evan }}= & \frac{\hbar}{4 \pi^{3}} \int_{0}^{\infty} d \omega\left(-\frac{\partial n}{\partial \omega}\right) \int_{q>\omega / c} d^{2} \mathbf{q}|\gamma|^{2} \times\left[\left(\operatorname{Im} \frac{2 R_{1 p} R_{2 p} e^{-2|\gamma| d}-R_{1 p}-R_{2 p} e^{-2|\gamma| d}}{1-e^{-2|\gamma| d} R_{1 p} R_{2 p}}\right)^{2}+\left(\operatorname{Im} \frac{2 R_{1 p} R_{2 p} e^{-2|\gamma| d}+R_{1 p}+R_{2 p} e^{-2|\gamma| d}}{1-e^{-2|\gamma| d} R_{1 p} R_{2 p}}\right)^{2}\right. \\
& \left.-2\left(\operatorname{Im} \frac{R_{1 p}-R_{2 p} e^{-2|\gamma| d}}{1-e^{-2|\gamma| d} R_{1 p} R_{2 p}}\right)^{2}+[p \rightarrow s]\right]=\frac{\hbar}{\pi^{2}} \int_{0}^{\infty} d \omega\left(-\frac{\partial n}{\partial \omega}\right) \int_{\omega / c}^{\infty} d q q|\gamma|^{2} e^{-2|\gamma| d} \\
& \times\left[\left(\operatorname{Im} R_{1 p}+e^{-2|\gamma| d \mid}\left|R_{1 p}\right|^{2} \operatorname{Im} R_{2 p}\right)\left(\operatorname{Im} R_{2 p}+e^{-2|\gamma| d \mid}\left|R_{2 p}\right|^{2} \operatorname{Im} R_{1 p}\right)+e^{-2|\gamma| d \mid}\left(\operatorname{Im}\left(R_{1 p} R_{2 p}\right)\right)^{2}\right] \frac{1}{\left|1-e^{-2|\gamma| d} R_{1 p} R_{2 p}\right|^{4}}+[p \rightarrow s] .
\end{aligned}
$$

Equations (24) and (25) were first presented without derivation in Ref. 9. In Ref. 10, Eqs. (24) and (25) were derived using the dynamical modification of the semiclassical Lifshitz theory ${ }^{19}$ of the van der Waals interaction, and the Rytov theory ${ }^{41-43}$ of the fluctuating electromagnetic field.

\section{VAN DER WAALS FRICTION BETWEEN A SMALL PARTICLE AND PLANE SURFACE}

\section{A. Parallel relative motion}

For parallel relative motion the friction coefficient $\Gamma_{\|}$ $=\Gamma_{x x}=\Gamma_{y y}$. The Lorentz force acting on a small particle located at point $\mathbf{r}_{0}$ can be written in the form

$$
\hat{F}_{x}=\left[p_{k} \frac{\partial}{\partial x_{k}} E_{x}(\mathbf{r})+\frac{1}{c}\left(j_{y} B_{z}-j_{z} B_{y}\right)\right]_{\mathbf{r}=\mathbf{r}_{0}},
$$

where $\mathbf{p}$ and $\mathbf{j}$ are the dipole moment and current operators of the particle, respectively. $\mathbf{E}$ and $\mathbf{B}$ are the external electric and magnetic induction field operators, respectively. The interaction of the electromagnetic field with the particle is described by the Hamiltonian

$$
H_{\text {int }}=-\frac{1}{c} \mathbf{A}\left(\mathbf{r}_{0}\right) \cdot \mathbf{j}
$$

where $\mathbf{A}(\mathbf{r})$ is the vector potential operator. Taking into account that

$$
\begin{gathered}
\mathbf{j}=\frac{\partial}{\partial t} \mathbf{p}, \\
\boldsymbol{\nabla} \times \mathbf{E}=\frac{1}{c} \frac{\partial}{\partial t} \mathbf{B}
\end{gathered}
$$

one can prove that the friction coefficient is determined by Eq. (4), where

$$
\begin{aligned}
G_{x x}^{R}(\omega)= & \frac{i}{\hbar} \int_{0}^{\infty} d t e^{i \omega t} \times\left\langle p_{k}(t) \frac{\partial}{\partial x} E_{k}(\mathbf{r}, t) p_{l}(0) \frac{\partial}{\partial x^{\prime}} E_{l}\left(\mathbf{r}^{\prime}, 0\right)\right. \\
& \left.-p_{l}(0) \frac{\partial}{\partial x^{\prime}} E_{l}\left(\mathbf{r}^{\prime}, 0\right) p_{k}(t) \frac{\partial}{\partial x} E_{k}(\mathbf{r}, t)\right\rangle_{\mathbf{r}=\mathbf{r}^{\prime}=\mathbf{r}_{0}},
\end{aligned}
$$

where summation over repeated indexes is assumed. Performing similar calculations as in Sec. III and using the relation

$$
\mathbf{E}(\mathbf{r}, t)=-\frac{1}{c} \frac{\partial}{\partial t} \mathbf{A}(\mathbf{r}, t)
$$

gives

$$
\begin{aligned}
\Gamma_{\|}= & \frac{2 \hbar}{\pi} \int_{0}^{\infty} d \omega\left(-\frac{\partial n}{\partial \omega}\right)\left\{\sum_{k=x, y, z} \operatorname{Im} \alpha_{k k} \frac{\partial^{2}}{\partial x \partial x^{\prime}} \operatorname{Im} D_{k k}\left(\mathbf{r}, \mathbf{r}^{\prime}, \omega\right)\right. \\
& \left.-2 \operatorname{Re}\left[\alpha_{x x}(\omega) \alpha_{z z}^{*}(\omega)\right]\left(\frac{\partial}{\partial x} \operatorname{Im} D_{x z}\left(\mathbf{r}, \mathbf{r}_{0}, \omega\right)\right)^{2}\right\}_{\mathbf{r}=\mathbf{r}^{\prime}=\mathbf{r}_{0}},
\end{aligned}
$$

where $D_{i j}\left(\mathbf{r}, \mathbf{r}^{\prime}\right)$ are the Green's functions of the electromagnetic field for one plane surface. These Green functions can be obtained from the Green functions for the two-plane surface geometry (see Appendix A) by putting $R_{2 p(s)}=0$. The polarizability of the particle

$$
\alpha_{k k}(\omega)=\frac{i}{\hbar} \int_{0}^{\infty} d t e^{i \omega t}\left\langle p_{k}(t) p_{k}(0)-p_{k}(0) p_{k}(t)\right\rangle
$$

can be written as

$$
\alpha_{i i}(\omega)=\frac{\alpha_{i i}^{0}(\omega)}{1-\alpha_{i i}^{0}(\omega) D_{i i}\left(\mathbf{r}_{0}, \mathbf{r}_{0}\right)},
$$

where $\alpha_{i i}^{0}(\omega)$ is the free-particle polarizability. While deriving Eq. (32) we also used the identity

$$
\begin{gathered}
\operatorname{Im} \alpha_{x x}(\omega) \operatorname{Im}\left[\alpha_{z z}(\omega) \frac{\partial}{\partial x} D_{x z}\left(\mathbf{r}, \mathbf{r}_{0}, \omega\right) \frac{\partial}{\partial x} D_{x z}\left(\mathbf{r}, \mathbf{r}_{0}, \omega\right)\right]+\operatorname{Im} \alpha_{z z}(\omega) \operatorname{Im}\left[\alpha_{x x}(\omega) \frac{\partial}{\partial x} D_{x z}\left(\mathbf{r}, \mathbf{r}_{0}, \omega\right) \frac{\partial}{\partial x} D_{x z}\left(\mathbf{r}, \mathbf{r}_{0}, \omega\right)\right] \\
-2 \operatorname{Im}\left[\alpha_{x x}(\omega) \frac{\partial}{\partial x} D_{x z}\left(\mathbf{r}, \mathbf{r}_{0}, \omega\right)\right] \operatorname{Im}\left[\alpha_{z z}(\omega) \frac{\partial}{\partial x} D_{x z}\left(\mathbf{r}, \mathbf{r}_{0}, \omega\right)\right]=2 \operatorname{Re}\left[\alpha_{x x}(\omega) \alpha_{z z}^{*}(\omega)\right]\left(\frac{\partial}{\partial x} \operatorname{Im} D_{x z}\left(\mathbf{r}, \mathbf{r}_{0}, \omega\right)\right)^{2} \\
205413-6
\end{gathered}
$$




\section{B. Normal relative motion}

The friction coefficient for a particle moving normal to the sample surface can be obtained from calculations very similar to those for parallel relative motion. In this case the Green's function $G_{x x}^{R}$ must be replaced by $G_{z z}^{R}$, where

$$
\begin{aligned}
G_{z z}^{R}(\omega)= & \frac{i}{\hbar} \int_{0}^{\infty} d t e^{i \omega t} \times\left\langle p_{k}(t) \frac{\partial}{\partial z} E_{k}(\mathbf{r}, t) p_{l}(0) \frac{\partial}{\partial z^{\prime}} E_{l}\left(\mathbf{r}^{\prime}, 0\right)\right. \\
& \left.-p_{l}(0) \frac{\partial}{\partial z^{\prime}} E_{l}\left(\mathbf{r}^{\prime}, 0\right) p_{k}(t) \frac{\partial}{\partial z} E_{k}(\mathbf{r}, t)\right\rangle_{\mathbf{r}=\mathbf{r}^{\prime}=\mathbf{r}_{0}}
\end{aligned}
$$

Performing similar calculations as in Sec. IV A we get

$$
\begin{aligned}
\Gamma_{\perp}= & \frac{2 \hbar}{\pi} \int_{0}^{\infty} d \omega\left(-\frac{\partial n}{\partial \omega}\right) \\
& \times \sum_{k=x, y, z}\left\{\operatorname { I m } \alpha _ { k k } ( \omega ) \frac { \partial ^ { 2 } } { \partial z \partial z ^ { \prime } } \left\{\operatorname{Im} D_{k k}\left(\mathbf{r}, \mathbf{r}^{\prime}, \omega\right)\right.\right. \\
& \left.+\operatorname{Im}\left[\alpha_{k k} D_{k k}\left(\mathbf{r}, \mathbf{r}_{0}, \omega\right) D_{k k}\left(\mathbf{r}^{\prime}, \mathbf{r}_{0}, \omega\right)\right]\right\} \\
& \left.+\left[\frac{\partial}{\partial z} \operatorname{Im}\left[\alpha_{k k}(\omega) D_{k k}\left(\mathbf{r}, \mathbf{r}_{0}, \omega\right)\right]\right]^{2}\right\}
\end{aligned}
$$

For a spherical particle with radius $R$, Eq. (37) is only valid if $R \ll d$. In the nonresonant case $\alpha_{k k}^{0} \sim R^{3}$ and $D_{k k} \sim d^{-3}$. Thus in this case $\alpha D \sim(R / d)^{3} \ll 1$ and we can neglect the screening effects. For a spherical particle $\alpha_{k k}=\alpha$, and using the formula (which is valid in the nonretarded limit, formally obtained as $c \rightarrow \infty$, see Ref. 27 and also Appendix A)

$$
\begin{aligned}
\sum_{k=x, y, z} D_{k k}\left(\mathbf{r}, \mathbf{r}^{\prime}, \omega\right)= & 4 \pi \int \frac{d^{2} q q}{(2 \pi)^{2}}\left[e^{-q\left|z-z^{\prime}\right|}\right. \\
& \left.+R_{p}(q, \omega) e^{-q\left(z+z^{\prime}\right)}\right] e^{i \mathbf{q}\left(\mathbf{x}-\mathbf{x}^{\prime}\right)}
\end{aligned}
$$

we get from Eqs. (32) and (37)

$$
\Gamma_{\|}=2 \frac{\hbar}{\pi} \int_{0}^{\infty} d \omega\left(-\frac{\partial n(\omega)}{\partial \omega}\right) \int_{0}^{\infty} d q q^{4} e^{-2 q d} \operatorname{Im} R_{p}(q, \omega) \operatorname{Im} \alpha(\omega)
$$

and $\Gamma_{\perp}=2 \Gamma_{\|}$. Equations (32) and (37) were obtained in Ref. 27 using the dynamical modification of the semiclassical Rytov theory ${ }^{41-43}$ of the fluctuating electromagnetic field, and Eq. (39) was derived in Ref. 33 using the fluctuationdissipation theorem.

\section{VAN DER WAALS FRICTION BETWEEN DIELECTRICS AND TWO-DIMENSIONAL SYSTEMS}

In Refs. 44 and 10 it was shown that the van der Waals friction between good conductors $\left(k_{B} T / 4 \pi \hbar \sigma \ll 1\right.$, where $\sigma$ is the conductivity) is extremely small. However the van der Waals friction can be greatly enhanced for high resistivity materials $\left(k_{B} T / 4 \pi \hbar \sigma>1\right)$. Thus, for two surfaces in parallel relative motion, using the reflection amplitude in the electrostatic limit $\left[d<c\left(\hbar / 4 \pi \sigma k_{B} T\right)^{1 / 2}\right]$

$$
R_{p}=\frac{\varepsilon-1}{\varepsilon+1}
$$

we get from Eq. (17)

$$
\gamma_{\|} \approx 0.05 \frac{\hbar}{d^{4}} \frac{k_{B} T}{4 \pi \hbar \sigma}
$$

and from Eq. (35), $\gamma_{\perp} \approx 10 \gamma_{\|}$. However, the macroscopic theory which was used in obtaining Eq. (40) is only valid when the average separation between the conduction electrons in the solids is much smaller than the length scale of variation of the electric field, which is determined by the separation $d$. Thus the lowest value of the conduction electron concentration is $n_{\text {min }} \sim d^{-3}$ and according to Drude formula the lowest value of the conductivity is $\sigma_{\min } \sim e^{2} \tau / m d^{3}$. Thus, the maximum of the friction, for which the macroscopic theory is valid, can be estimated as

$$
\gamma_{\| \max } \sim 0.05 \frac{\hbar}{d^{4}} \frac{k_{B} T}{4 \pi \hbar \sigma_{\min }} \sim 0.05 \frac{m k_{B} T}{4 \pi e^{2} \tau d} .
$$

The friction coefficient $\Gamma$ for an atomic force microscope tip with the radius of curvature $R \gg d$ can be estimated using the "proximity approximation."45,34 This approximation was proposed for the van der Waals interaction but there is no reason why this approximation will not be valid for the van der Waals friction. In this approximation the tip and sample are treated as a set of piecewise parallel planes. The accuracy of this scheme was checked in Ref. 34. Thus for the friction coefficient for a spherical tip we get

$$
\Gamma_{\|}^{s} \approx 2 \pi \int_{0}^{\infty} d \rho \rho \gamma_{\|}\left(d+\rho^{2} / 2 R\right)=0.05 \frac{2 \pi R}{3 d^{3}} \frac{k_{B} T}{4 \pi \sigma}
$$

and the maximum of friction can be estimated as

$$
\Gamma_{\| \max }^{s} \sim 0.05 \frac{2 \pi R}{3 d^{3}} \frac{k_{B} T}{4 \pi \sigma_{\min }} \sim 0.1 \frac{m k_{B} T R}{4 \pi e^{2} \tau} .
$$

For $\tau \sim 10^{-16} \mathrm{~s}, R \sim 1 \mu \mathrm{m}$, and $T=300 \mathrm{~K}$ we get $\Gamma_{\max }$ $\sim 10^{-15} \mathrm{~kg} / \mathrm{s}$. This friction is two orders of magnitude smaller than the friction observed in a recent experiment at $d=10 \mathrm{~nm} .{ }^{6}$ Similarly, in the case of a cylindrical tip we get

$$
\Gamma_{\|}^{c} \approx 2 w \int_{0}^{\infty} d \rho \gamma_{\|}\left(d+\rho^{2} / 2 R\right)=\frac{2^{1 / 2} \pi}{64} \sqrt{\frac{R}{d}} \frac{k_{B} T w}{4 \pi \sigma d^{3}},
$$

where $w$ is the width of the tip, and the maximum of friction can be estimated as

$$
\Gamma_{\| \max }^{c} \sim \frac{2^{1 / 2} \pi}{64} \sqrt{\frac{R}{d}} \frac{k_{B} T w}{4 \pi \sigma_{\min } d^{3}} \sim \frac{2^{1 / 2} \pi}{64} \sqrt{\frac{R}{d}} \frac{m k_{B} T w}{4 \pi e^{2} \tau} .
$$

For $w=7 \mu \mathrm{m}, d=10 \mathrm{~nm}$, and with the other parameters as above, Eq. (46) gives friction of the same order of magnitude as it was observed in the experiment. ${ }^{6}$

Recently a large electrostatic noncontact friction has been observed between an atomic force microscope tip and thin dielectric films. ${ }^{13}$ The van der Waals friction will also be large for dielectrics with high absorption at low frequencies. 


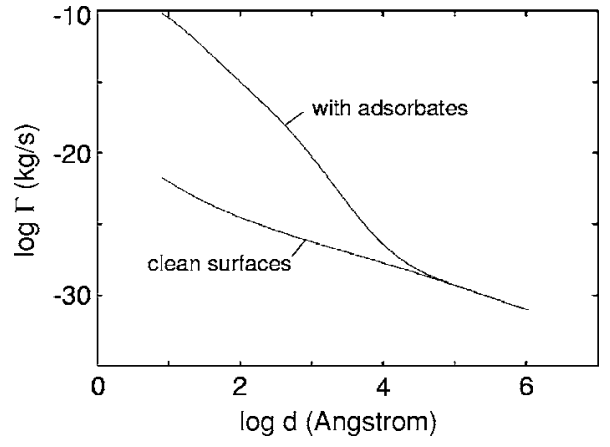

FIG. 2. The friction coefficient associated with the van der Waals friction between a copper tip and a copper substrate, both covered by low concentration of cesium atoms, as a function of the separation $d$. The cylindrical tip is characterized by the radius of curvature $R=1 \mu \mathrm{m}$ and the width $w=7 \mu \mathrm{m}$. The other parameters correspond to $\mathrm{Cs}$ adsorbed on $\mathrm{Cu}(100)$ at the concentration $n_{a}$ $=10^{18} \mathrm{~m}^{-2}$ (coverage $\left.\theta \approx 0.1\right)$ (Refs. 11 and 48): $e^{*}=0.28 e, \eta$ $=10^{11} \mathrm{~s}^{-1}, a=2.94 \AA, T=293 \mathrm{~K}$. (The base of the logarithm is 10.)

As an example we consider the van der Waals friction between thin water films adsorbed on transparent dielectric substrates such as silica or mica. Water has an extremely large static dielectric function of around 80. The low frequency contribution to the dielectric function, responsible for this large static value, is due to relaxation of the permanent dipoles of the water molecules. It can be accurately described by the Debye ${ }^{46}$ theory of rotational relaxation. The theoretical fit of the experimental data is given by ${ }^{47}$

$$
\varepsilon(\omega)=4.35+\frac{C}{1-i \omega / \omega_{0}},
$$

where $C=72.24$ and $\omega_{0}=1.3 \times 10^{11} \mathrm{~s}^{-1}$. We note that water has large absorption in the radio-frequency range at $\omega \sim \omega_{0}$, and shows in this region of the spectrum anomalous dispersion. In this frequency range the dielectric constants $\varepsilon_{2}$ of mica or silica are nearly constant (and real) and $\left|\varepsilon_{3}\right| \gg \varepsilon_{2}$, where $\varepsilon_{3}$ denotes the dielectric function of water. For a planar film with thickness $h$ and dielectric function $\varepsilon_{3}(\omega)$ on top of a substrate with the dielectric function $\varepsilon_{2}(\omega)$ the reflection amplitude

$$
R_{p}=\frac{R_{p 31}-R_{p 32} \exp (-2 q h)}{1-R_{p 31} R_{p 32} \exp (-2 q h)},
$$

where

$$
R_{p i j}=\frac{\varepsilon_{i}-\varepsilon_{j}}{\varepsilon_{i}+\varepsilon_{j}} .
$$

For $q h \ll 1$ and $q^{-1} \sim d \ll\left|\varepsilon_{3}\right| h / \varepsilon_{2}$ the reflection amplitude can be approximated by

$$
R_{p} \approx 1-\frac{2}{\varepsilon_{3} q h} .
$$

Substituting Eq. (50) into Eq. (17) and using the "proximity approximation" we obtain the friction between a cylindrical atomic force microscope tip and a sample

$$
\Gamma_{\|}^{c}=\frac{\pi \hbar R^{1 / 2} w}{6 \sqrt{2} C^{2} h^{2} d^{3 / 2}}\left(\frac{k_{B} T}{\hbar \omega_{0}}\right)^{2} .
$$

For $h=1 \mathrm{~nm}$ and with the other parameters the same as above we get $\Gamma_{\|}^{c}=4.8 \times 10^{-12} \mathrm{~kg} / \mathrm{s}$. The friction observed in Ref. 6 has the same weak distance dependence as predicted by Eq. (51), but is one order of magnitude smaller than predicted by Eq. (51).

Another enhancement mechanism of the van der Waals friction is connected with resonant photon tunneling between adsorbate vibrational modes localized on different surfaces. In Refs. 9 and 10 we have shown that resonant photon tunneling between two surfaces separated by $d=1 \mathrm{~nm}$, and covered by a low concentration of potassium atoms, result in a friction which is six orders of the magnitude larger than for clean surfaces. The adsorbate induced enhancement of the van der Waals friction is even larger for Cs adsorption on $\mathrm{Cu}(100)$. In this case, even at low coverage $(\theta \sim 0.1)$, the adsorbed layer exhibits an acoustic branch for vibrations parallel to the surface, ${ }^{48}$ and according to Ref. 12 , at small frequencies the reflection amplitude is given by Ref. 12

$$
R_{p}=1-\frac{2 q a \omega_{q}^{2}}{\omega^{2}-\omega_{q}^{2}+i \omega \eta},
$$

where $\omega_{q}^{2}=4 \pi n_{a} e^{* 2} a q^{2} / M, e^{*}$ is the ion charge and $a$ is the separation between an ion and the image plane. Substituting Eq. (52) in Eq. (17), and assuming that

$$
\frac{a}{\eta d} \sqrt{\frac{4 \pi n_{a} e^{* 2} a}{M d^{2}}} \ll 1,
$$

and using the "proximity approximation," for a cylindrical tip we get

$$
\Gamma_{\|}^{c} \approx 0.68 \frac{k_{B} T a^{2} R^{0.5} w}{\eta d^{5.5}} .
$$

For $\mathrm{Cs}$ adsorption on $\mathrm{Cu}(100)$ the damping parameter $\eta$ was estimated in Ref. 12 as $\eta \approx 10^{11} \mathrm{~s}^{-1}$. Using this value of $\eta$ in Eq. (53) for $a=2.94 \AA{ }^{48} R=1 \mu \mathrm{m}, w=7 \mu \mathrm{m}, T=293 \mathrm{~K}$ at $d=10 \mathrm{~nm}$ we get $\Gamma_{\|} \approx 10^{-15} \mathrm{~kg} / \mathrm{s}$, which is two orders of magnitude smaller than the friction observed in Ref. 6 at the same distance. However, in this case the van der Waals friction is characterized by a much stronger distance dependence $\left(\sim 1 / d^{5.5}\right)$ than observed in the experiment $\left(\sim 1 / d^{n}\right.$, where $n=1.3 \pm 0.2$ ). Thus, at small distances the van derWaals friction will be larger than friction observed in 6 , and can thus be measured experimentally. Figure 2 shows how the friction between the copper tip and the copper substrate depends on the distance $d$ for clean surfaces, and when the surfaces of the tip and the substrate are covered by a low concentration of the Cs atoms. In comparison with the adsorbate covered surfaces, the friction between two clean surfaces at the separation $d=1 \mathrm{~nm}$ is eleven orders of the magnitude smaller. However, the friction between clean surfaces shown in Fig. 2 was calculated using the local optic approximation, where the spatial variation of the dielectric function is neglected. For parallel relative motion nonlocal optic effects are very important, ${ }^{10}$ and when it is taken into account, at $d=1 \mathrm{~nm}$ the 
friction between the adsorbate covered surfaces will be seven orders of the magnitude larger than the friction between clean surfaces.

\section{SUMMARY AND CONCLUSION}

We have used thermal quantum field theory in the Matsubara formulation to calculate the van der Waals friction between two plane parallel surfaces, and between a small particle and plane surface, for both parallel and normal relative motion. The friction calculated in this approach agrees with the friction calculated using a dynamical modification of the Lifshitz theory, which is based on the fluctuationdissipation theorem. In quantum field theory the calculation of the friction to linear order in the sliding velocity is reduced to finding the equilibrium Green functions which obey Maxwell-type equations. Thus, using numerical methods developed for classical electrodynamics, this approach can be used to obtain the van der Waals friction between bodies with complex geometry. We have shown that the van der Waals friction between high-resistivity metals, dielectrics with strong absorption in radio-frequency range, and twodimensional systems can be measured in noncontact friction experiments using state-of-the-art equipment. The theory can be used as a guide for designing and interpreting.

\section{ACKNOWLEDGMENTS}

A.I.V acknowledges financial support from Russian Foundation for Basic Research (Grant No. 06-02-16979), DFG, and ESF project "Nanotribology."

\section{APPENDIX A: THE GREEN FUNCTIONS FOR TWO PLANE SURFACES}

Suppose that the half-space $z<0$ is occupied by a solid with the reflection amplitude $R_{1 p}(\mathbf{q}, \omega)$ and $R_{1 s}(\mathbf{q}, \omega)$ for $p$ and $s$-electromagnetic fields, respectively. Similarly, the halfspace $z>d$ is occupied with a solid with the reflection amplitude $R_{2 p}(\mathbf{q}, \omega)$ and $R_{2 s}(\mathbf{q}, \omega)$. The region $0<z<d$ is assumed to be vacuum. Here $\mathbf{q}$ is the surfaces component of the wave vector $\mathbf{k}=(\mathbf{q}, \gamma)$, where $\gamma=\left[(\omega / c)^{2}-q^{2}\right]^{1 / 2}$. Since the system is homogeneous in the $\mathbf{x}=(x, y)$ plane, the Green function $D_{i j}\left(\mathbf{r}, \mathbf{r}^{\prime}\right)$ can be represented by the Fourier integral

$$
D_{i j}\left(\mathbf{r}, \mathbf{r}^{\prime}\right)=\int \frac{d^{2} q}{(2 \pi)^{2}} e^{i \mathbf{q} \cdot\left(\mathbf{x}-\mathbf{x}^{\prime}\right)} D_{i j}\left(z, z^{\prime}, \mathbf{q}\right) .
$$

In the $x y$ plane it is convenient to choose the coordinate axes along the vectors $\hat{q}=\mathbf{q} / q$ and $\hat{n}=\hat{z} \times \hat{q}$. In this coordinate system Eqs. (10) and (11) for the Green functions become

$$
\begin{gathered}
\left(\gamma^{2}+\frac{\partial^{2}}{\partial z^{2}}\right) D_{n n}\left(z, z^{\prime}\right)=-\frac{4 \pi \omega^{2}}{c^{2}} \delta\left(z-z^{\prime}\right), \\
\left(\frac{\omega^{2}}{c^{2}}-\frac{\partial^{2}}{\partial z^{2}}\right) D_{q q}\left(z, z^{\prime}\right)-i q \frac{\partial}{\partial z} D_{z q}\left(z, z^{\prime}\right)=-\frac{4 \pi \omega^{2}}{c^{2}} \delta\left(z-z^{\prime}\right),
\end{gathered}
$$

$$
\begin{gathered}
\gamma^{2} D_{z q}\left(z, z^{\prime}\right)-i q \frac{\partial}{\partial z} D_{q q}\left(z, z^{\prime}\right)=0, \\
\gamma^{2} D_{z z}\left(z, z^{\prime}\right)-i q \frac{\partial}{\partial z} D_{q z}\left(z, z^{\prime}\right)=-\frac{4 \pi \omega^{2}}{c^{2}} \delta\left(z-z^{\prime}\right), \\
\gamma^{2} D_{q z}\left(z, z^{\prime}\right)+i q \frac{\partial}{\partial z^{\prime}} D_{q q}\left(z, z^{\prime}\right)=0 .
\end{gathered}
$$

The equations for $D_{q n}$ and $D_{z n}$ turn out to be homogeneous so these Green functions vanish. Solving the system of equations (A2)-(A6) amounts to solving two equations: Eq. (A2) for $D_{n n}$, and the equation for $D_{q q}$ which follows from Eqs. (A3) and (A4)

$$
\left(\gamma^{2}+\frac{\partial^{2}}{\partial z^{2}}\right) D_{q q}\left(z, z^{\prime}\right)=-4 \pi \gamma^{2} \delta\left(z-z^{\prime}\right) .
$$

$D_{q z}, D_{z q}$, and $D_{z z}$ for $z \neq z^{\prime}$ are obtained from Eqs. (A4)-(A6) as

$$
\begin{gathered}
D_{q z}^{R}=-\frac{i q}{\gamma^{2}} \frac{\partial}{\partial z^{\prime}} D_{q q}, \quad D_{z q}=\frac{i q}{\gamma^{2}} \frac{\partial}{\partial z} D_{q q}, \\
D_{z z}=\frac{q^{2}}{\gamma^{4}} \frac{\partial^{2}}{\partial z \partial z^{\prime}} D_{q q} .
\end{gathered}
$$

In the vacuum gap $0<z<d$ the solution of equation (A2) has the form

$$
D_{n n}\left(z, z^{\prime}\right)=-\frac{2 \pi i \omega^{2}}{\gamma c^{2}} e^{i \gamma\left|z-z^{\prime}\right|}+v_{n} e^{i \gamma z}+w_{n} e^{-i \gamma z} .
$$

At the boundaries $z=0$ and $z=d$ the amplitude of the reflected wave is equal to the amplitude of incident wave times to the corresponding reflection amplitude. The Green function $D_{n n}$ is associated with the $s$-polarized electromagnetic field, and the boundary conditions for it gives

$$
\begin{gathered}
v_{n}=R_{1 s}\left(w_{n}+\frac{2 \pi i \omega^{2}}{\gamma c^{2}} e^{i \gamma z^{\prime}}\right) \quad \text { for } z=0, \\
w_{n}=R_{2 s} e^{2 i \gamma d}\left(v_{n}+\frac{2 \pi i \omega^{2}}{\gamma c^{2}} e^{-i \gamma z^{\prime}}\right) \quad \text { for } z=d .
\end{gathered}
$$

Using Eqs. (A10)-(A12) we get

$$
\begin{aligned}
D_{n n}\left(z, z^{\prime}\right)= & -\frac{2 \pi i \omega^{2}\left\{e^{i \gamma\left|z-z^{\prime}\right|}\right.}{\gamma c^{2}} \\
& +\frac{R_{1 s} R_{2 s} e^{2 i \gamma d}\left(e^{i \gamma\left(z-z^{\prime}\right)}+e^{-i \gamma\left(z-z^{\prime}\right)}\right)}{\Delta_{s}} \\
& \left.+\frac{R_{1 s} e^{i \gamma\left(z+z^{\prime}\right)}+R_{2 s} e^{2 i \gamma d} e^{-i \gamma\left(z+z^{\prime}\right)}}{\Delta_{s}}\right\}, \\
& \Delta_{s}=1-e^{2 i \gamma d} R_{2 s} R_{1 s} .
\end{aligned}
$$

Equation (A7) for $D_{q q}$ is similar to Eq. (A2) for $D_{n n}$, and the expression for $D_{q q}^{R}$ can be obtained from expression (A13) by replacements of the reflection amplitude 


$$
D_{q q}=\left(\frac{\gamma c}{\omega}\right)^{2} D_{n n}\left[R_{s} \rightarrow-R_{p}\right] .
$$

The calculation of the reflection amplitude for $s$ - and $p$-polarized waves constitutes separate problems, which can be solved taking into account nonlocal effects. For the local optic case the reflection amplitude are determined by the well known Fresnel formulas

$$
R_{i p}=\frac{\varepsilon_{i} \gamma-\gamma_{i}}{\varepsilon_{i} \gamma+\gamma_{i}}, \quad R_{i s}=\frac{\gamma-\gamma_{i}}{\gamma+\gamma_{i}},
$$

where $\varepsilon_{i}$ is the complex dielectric constant for body $i$ :

$$
\begin{aligned}
& \qquad \gamma_{i}=\sqrt{\frac{\omega^{2}}{c^{2}} \varepsilon_{i}-q^{2}} . \\
& \text { APPENDIX B: FREQUENCY SUMMATIONS }
\end{aligned}
$$

\section{APPENDIX B: FREQUENCY SUMMATIONS}

All the sums over $\omega_{m}$ in Eq. (13) can be calculated in a similar way. Thus, as an illustration, we consider only one sum

$$
\frac{1}{\beta} \sum_{\omega_{m}} D_{q z}^{E E}\left(\mathbf{q}, i \omega_{m}\right) D_{z q}^{E E}\left(-\mathbf{q}, i \omega_{n}-i \omega_{m}\right)
$$

According to the Lehmann representation, the Green's function can be written in the form

$$
D_{\alpha \beta}^{E E}\left(\omega_{n}, \mathbf{r}, \mathbf{r}^{\prime}\right)=\frac{1}{\pi} \int_{-\infty}^{\infty} d x \frac{\rho_{\alpha \beta}^{E E}\left(x, \mathbf{r}, \mathbf{r}^{\prime}\right)}{x-i \omega_{n}},
$$

where

$$
\begin{aligned}
\rho_{\alpha \beta}^{E E}\left(\omega, \mathbf{r}, \mathbf{r}^{\prime}\right)= & \sum_{n, m} \exp \left(F-E_{n}\right)\left[E_{\alpha}(\mathbf{r})\right]_{n m}\left[E_{\alpha}\left(\mathbf{r}^{\prime}\right)\right]_{m n} \\
& \times\left(1-e^{-\beta \omega_{m n}}\right) \delta\left(\omega-\omega_{m n}\right) .
\end{aligned}
$$

Using Eq. (B2) and standard rules for the evaluation of a sum such as Eq. (B1) (Ref. 40) we get

$$
\begin{aligned}
& \frac{1}{\beta} \sum_{\omega_{m}} D_{q z}^{E E}\left(\mathbf{q}, \omega_{m}\right) D_{z q}^{E E}\left(-\mathbf{q}, i \omega_{n}-i \omega_{m}\right) \\
& =\int_{-\infty}^{\infty} d \omega\left\{\left[\rho_{q z}^{E E}(\mathbf{q}, \omega) D_{z q}^{E E}\left(-\mathbf{q}, i \omega_{n}-\omega\right)\right] n(\omega)+\left[D _ { q z } ^ { E E } \left(\mathbf{q}, i \omega_{n}\right.\right.\right. \\
& \left.\left.\quad-\omega) \rho_{z q}^{E E}(-\mathbf{q}, \omega)\right][n(\omega)+1]\right\},
\end{aligned}
$$

where $n(\omega)=\left[\exp \left(\hbar \omega / k_{B} T\right)-1\right]^{-1}$. Using Eqs. (A8) and (A9) in (B3) we get

$$
\begin{aligned}
\frac{\hbar}{\beta} \sum_{\omega_{m}} D_{q z}^{E E}\left(\mathbf{q}, i \omega_{m}\right) D_{z q}^{E E}\left(-\mathbf{q}, i \omega_{n}-i \omega_{m}\right) \\
=-q^{2} \frac{\hbar}{\pi} \int_{-\infty}^{\infty} d \omega \\
\quad \times\left[\left(\frac{\partial}{\partial z^{\prime}} \frac{\rho_{q q}^{E E}\left(\omega, z, z^{\prime}\right)}{\gamma^{2}(\omega)} \frac{\partial}{\partial z} \frac{D_{q q}\left(i \omega_{n}-\omega, z, z^{\prime}\right)}{\gamma^{2}\left(i \omega_{n}-\omega\right)}\right) n(\omega)\right. \\
\left.\quad+\left(\frac{\partial}{\partial z^{\prime}} \frac{D_{q q}^{E E}\left(i \omega_{n}-\omega, z, z^{\prime}\right)}{\gamma^{2}\left(i \omega_{n}-\omega\right)} \frac{\partial}{\partial z} \frac{\rho_{q q}^{E E}\left(\omega, z, z^{\prime}\right)}{\gamma^{2}(\omega)}\right)[n(\omega)+1]\right],
\end{aligned}
$$

where $\gamma^{2}(\omega)=(\omega / c)^{2}-q^{2}$. Replacing $i \omega_{n} \rightarrow \omega_{0}+i \delta$ and taking the imaginary part of Eq. (B4) gives, in the limit $\omega_{0} \rightarrow 0$, the following contribution to the friction coming from Eq. (B4):

$$
\begin{aligned}
\lim _{\omega_{0} \rightarrow 0} & \frac{1}{\omega_{0}} \operatorname{Im}_{i \omega_{n} \rightarrow \omega_{0}+i \delta} \lim _{\beta} \frac{\hbar}{\beta} \sum_{\omega_{m}} D_{q z}^{E E}\left(\mathbf{q}, i \omega_{m}\right) D_{z q}^{E E}\left(-\mathbf{q}, i \omega_{n}-i \omega_{m}\right) \\
= & -\frac{2 \hbar q^{2}}{\pi \gamma^{4}} \int_{0}^{\infty} d \omega\left(-\frac{\partial n}{\partial \omega}\right)\left(\frac{\partial}{\partial z} \operatorname{Im} D_{q q}(\omega)\right) \\
& \times\left(\frac{\partial}{\partial z^{\prime}} \operatorname{Im} D_{q q}(\omega)\right) .
\end{aligned}
$$

*Email address: avoli@samgtu.ru

${ }^{1}$ T. J. Gramila, J. P. Eisenstein, A. H. MacDonald, L. N. Pfeiffer, and K. W. West, Phys. Rev. Lett. 66, 1216 (1991); Surf. Sci. 263, 446 (1992).

${ }^{2}$ T. J. Gramila, J. P. Eisenstein, A. H. MacDonald, L. N. Pfeiffer, and K. W. West, Phys. Rev. B 47, 12957 (1993); Physica B 197, 442 (1994).

${ }^{3}$ U. Sivan, P. M. Solomon, and H. Shtrikman, Phys. Rev. Lett. 68, 1196 (1992).

${ }^{4}$ I. Dorofeyev, H. Fuchs, G. Wenning, and B. Gotsmann, Phys. Rev. Lett. 83, 2402 (1999).

${ }^{5}$ B. Gotsmann and H. Fuchs, Phys. Rev. Lett. 86, 2597 (2001).

${ }^{6}$ B. C. Stipe, H. J. Mamin, T. D. Stowe, T. W. Kenny, and D. Rugar, Phys. Rev. Lett. 87, 096801 (2001).

${ }^{7}$ H. J. Mamin and D. Rugar, Appl. Phys. Lett. 79, 3358 (2001).

${ }^{8}$ P. M. Hoffmann, S. Jeffery, J. B. Pethica, H. Özgür Özer, and A.
Oral, Phys. Rev. Lett. 87, 265502 (2001).

${ }^{9}$ A. I. Volokitin and B. N. J. Persson, Phys. Rev. Lett. 91, 106101 (2003).

${ }^{10}$ A. I. Volokitin and B. N. J. Persson, Phys. Rev. B 68, 155420 (2003).

${ }^{11}$ A. I. Volokitin and B. N. J. Persson, Phys. Rev. Lett. 94, 086104 (2005).

${ }^{12}$ A. I. Volokitin, B. N. J. Persson, and H. Ueba, Phys. Rev. B 73, 165423 (2006).

${ }^{13}$ S. Kuehn, R. F. Loring, and J. A. Marohn, Phys. Rev. Lett. 96, 156103 (2006).

${ }^{14}$ D. Rugar, R. Budakian, H. J. Mamin, and B. W. Chui, Nature (London) 430, 329 (2004).

${ }^{15}$ J. A. Sidles, J. L. Carbini, K. J. Bruland, D. Rugar, O. Zuger, S. Hoen, and C. S. Yannoni, Rev. Mod. Phys. 67, 249 (1995).

${ }^{16}$ G. P. Berman, G. D. Doolen, P. C. Hammel, and V. I. Tsifrinovich, 
Phys. Rev. B 61, 14694 (2000).

${ }^{17}$ N. Arkani-Hamed, S. Dimopoulos, and G. Dvali, Phys. Lett. B 429, 263 (1998); Sci. Am. 283, 62 (2000).

${ }^{18}$ U. Mohideen and A. Roy, Phys. Rev. Lett. 81, 4549 (1998).

${ }^{19}$ E. M. Lifshitz, Zh. Eksp. Teor. Fiz. 29, 94 (1955) [Sov. Phys. JETP 2, 73 (1956)].

${ }^{20}$ I. E. Dzyaloshinskii, E. M. Lifshitz, and L. P. Pitaevskii, Adv. Phys. 10, 165 (1961).

${ }^{21}$ J. B. Pendry, J. Phys.: Condens. Matter 9, 10301 (1997).

${ }^{22}$ A. I. Volokitin and B. N. J. Persson, J. Phys.: Condens. Matter 11, 345 (1999); Phys. Low-Dimens. Struct. 7/8, 17 (1998).

${ }^{23}$ E. V. Teodorovitch, Proc. R. Soc. London, Ser. A 362, 71 (1978).

${ }^{24}$ J. Mahanty, J. Phys. B 13, 4391 (1980).

${ }^{25}$ G. V. Dedkov and A. A. Kyasov, Phys. Lett. A 259, 38 (1999).

${ }^{26}$ A. A. Kyasov and G. V. Dedkov, Surf. Sci. 463, 11 (2000).

${ }^{27}$ A. I. Volokitin and B. N. J. Persson, Phys. Rev. B 65, 115419 (2002).

${ }^{28}$ W. L. Schaich and J. Harris, J. Phys. F: Met. Phys. 11, 65 (1981).

${ }^{29}$ L. S. Levitov, Europhys. Lett. 8, 499 (1989).

${ }^{30}$ V. G. Polevoi, Zh. Eksp. Teor. Fiz. 98, 1990 (1990) [Sov. Phys. JETP 71, 1119 (1990)].

${ }^{31}$ V. E. Mkrtchian, Phys. Lett. A 207, 299 (1995).

${ }^{32}$ B. N. J. Persson and Z. Zhang, Phys. Rev. B 57, 7327 (1998).

${ }^{33}$ S. Tomassone and A. Widom, Phys. Rev. B 56, 4938 (1997).

${ }^{34}$ P. Johansson and P. Apell, Phys. Rev. B 56, 4159 (1997).

${ }^{35}$ A. A. Abrikosov, L. P. Gor'kov, and I. Ye. Dzyaloshinskii, Quantum Field Theoretical Methods in Statistical Physics (Pergamon
Press, Oxford, 1965).

${ }^{36}$ L. D. Landau and E. M. Lifschitz, Statistical Physics (Pergamon, Oxford, 1970).

${ }^{37}$ W. L. Schaich, J. Chem. Phys. 60, 1087 (1974); Solid State Commun. 15, 357 (1974).

${ }^{38}$ E. G. d'Agliano, P. Kumar, W. Schaich, and H. Suhl, Phys. Rev. B 11, 2122 (1975).

${ }^{39}$ A. Nourtier, J. Phys. (Paris) 38, 479 (1977).

${ }^{40}$ G. Mahan, Many-Particle Physics, 2nd ed. (Plenum Press, New York, 1990).

${ }^{41}$ S. M. Rytov, Theory of Electrical Fluctuation and Thermal Radiation (Academy of Science, Moscow, 1953).

${ }^{42}$ M. L. Levin and S. M. Rytov, Theory of Equilibrium Thermal Fluctuations in Electrodynamics (Science Publishing, Moscow, 1967).

${ }^{43}$ S. M. Rytov, Yu. A. Kravtsov, and V. I. Tatarskii, Principles of Statistical Radiophysics (Springer, New York, 1989), Vol. 3.

${ }^{44}$ B. N. J. Persson and A. I. Volokitin, Phys. Rev. Lett. 84, 3504 (2000).

${ }^{45}$ U. Hartmann, Phys. Rev. B 42, 1541 (1990); 43, 2404 (1991).

${ }^{46}$ P. Debye, Polar Molecules (Chemical Catalog Co., New York, 1929), Chap. V.

${ }^{47}$ B. E. Sernelius, Surface Modes in Physics (Wiley-VCH Verlag Berlin, Berlin, 2001), Chap. 2.

${ }^{48}$ P. Senet, J. P. Toennis, and G. Witte, Chem. Phys. Lett. 299, 389 (1999). 